\title{
THE AMMONIACAL NITROGEN OF PEATS AND HUMUS SOILS.
}

\section{PART I.}

By J. C. B. ELLIS, B.A., ANd C. G. T. MORISON, M.A. (School of Rural Economy, Oxford.)

THE detailed examination of humus soils of various kinds is becoming of increasing importance in view of the schemes now on foot for the reclamation of much heath and moor land. One of the most important things to establish has seemed to the authors to be the amount of nitrogen existing in the form of ammonia or of ammonium compounds, as it is possible that a knowledge of this figure might form a basis for a more scientific classification of soil organic matter than at present exists.

In those humus soils where so-called acid conditions obtain it might be expected that the amount of ammoniacal nitrogen would be high, as in these cases it would have the maximum opportunity for accumulation. Consequently the preliminary work was carried out with soils of this kind; mostly so-called acid peats.

It had previously been noticed by one of us that the method adopted by Russell ${ }^{1}$ for the determination of ammonia in arable soils seemed to give concordant results when applied to humus soils. The method consists essentially in the distillation of an adequate amount of the peat with magnesia at a temperature of $40^{\circ}$ under as low a pressure as can be obtained with an ordinary water pump.

It was decided to employ magnesia as the base for the liberation of ammonia, because the manipulation is easier, and because from Russell's results and from the results of our preliminary experiments there seemed no reason to doubt but that the magnesia was attacking a definite group of substances in the peat.

1 Morison and Sothers, This Journal, vol. 6, pt. 1, p. 88. 


\section{Ammoniacal Nitrogen of Peats and Humus Soils}

The actual details of the experiment are as follows.

The apparatus consisted of a round-bottomed litre flask fitted with a trap and another tube, the latter passing down to the bottom of the flask. This tube after leaving the flask was bent twice at right angles and tapered off into a fine capillary. The capillary was enclosed in a small bottle through the cork of which passed a small open tube. The bottle contained sulphuric acid, above the surface of which the capillary terminated. This device permitted of a slow stream of ammonia-free air passing into the flask during the distillation, in order to avoid bumping.

The trap in the distilling flask was connected by means of a glass tap to a 100 c.c. pipette which passed through a cork fitting into a filter flask containing sulphuric acid, into which the end of the pipette dipped.

The side-tube from the filter flask was connected to another glass tap, which was in turn connected to a water pump. Between the tap and the pump a manometer and safety bottle were inserted,

The glass taps were found necessary when it was decided to conduct three distillations in parallel, the three distilling flasks in the same water bath being connected to the same capillary, and the same water pump being used to evacuate the whole apparatus.

At the end of the distillation the glass taps on either side of each filter flask were turned off, and the pipette and flask disconnected for purposes of titration.

The preliminary determinations were made with air-dried peats, 30 grs. of which were found to be a convenient weight for purposes of distillation.

100 c.c. of $N / 100$ sulphuric acid were used in the filter flasks and, after the distillation, the excess of acid was determined by titration with $\mathrm{N} / 100$ potassium hydroxide, using methyl orange as indicator.

The method differs from that of Russell in that the distillation is only continued for three hours, and the liquid is not all distilled, a procedure which did not seem advisable in the case of a substance containing so much nitrogenous material as these soils.

In Table I are given the results obtained from the distillation of peat in the manner described for five hours, fresh acid being used every hour for the absorption of the ammonia given off.

The results are expressed as percentage of nitrogen in air-dried peat.

In every case a very marked drop occurs after the second hour, and after the third hour a small amount comes over which remains practically constant during the fourth and fifth hours. 
TABLE I.

$\begin{array}{lccc} & A & B & C \\ \text { Ist hour } & .00323 & .00390 & .00508 \\ \text { 2nd " } & .00508 & .00551 & .00529 \\ \text { 3rd " } & .00230 & .00230 & .00207 \\ \text { 4th " } & .00207 & .00184 & .00184 \\ \text { 5th " } & .00207 & .00162 & .00184\end{array}$

The ammonia which is most easily removable comes over in the first two hours. Distillations for three hours would make certain of the removal of the whole of the nitrogen arising from the decomposition of the more unstable compounds present, while as little as possible of the nitrogen from more stable compounds would be included in this result.

The nitrogen should come from ammonia present either as ammonium compounds and absorbed ammonia, or from organic compounds which are hydrolysed with comparative ease.

The amount of magnesia taken was fixed at three grams as a result of a set of determinations of which Table II contains examples.

These determinations were made by distilling the peat for three hours with 1 gram of magnesia, then adding a second gram and continuing the distillation for another three hours, and similarly for a third gram.

\section{TABLE II.}

\begin{tabular}{llll} 
& \multicolumn{3}{c}{ Nitrogen per cent. } \\
& Soil (1) & Soil (2) & Soil (3) \\
lst gr. MgO & .005866 & .007863 & .006878 \\
2nd $"$ & .001471 & .000475 & .000984 \\
3rd $"$ & .000992 & .000345 & .000051
\end{tabular}

Determinations were then made of the amounts of ammonia set free in this way from a series of humus soils.

The origin of the soils and the results are given in Table III.

It will be seen from these results that large quantities of ammonia may be set free from humus soils, which quantities are very much larger than the amounts set free from ordinary soils when treated in a similar way. To demonstrate this determinations were made in three ordinary soils:

(1) A coarse sand in rough permanent grass.

$\mathrm{N}$ as $\mathrm{NH}_{3}$, per cent.

(2) Arable soil on limestone gravel subsoil.

$\left\{\begin{array}{l}.00065 \\ .00060\end{array}\right.$

.00060

(.000்27

$\{.00037$

(3) Arable alluvial soil on gravel subsoil. $\left\{\begin{array}{l}.00046 \\ .00046\end{array}\right.$ 


\section{Ammoniacal Nitrogen of Peats and Humus Soils}

Table III.

Irish Peats :

Origin and Description

Shaughnessy's Bog. Old deposit of peat lying under permanent grass of fair quality. Carboniferous limestone. Altitude, $200 \mathrm{ft}$. S.W. Co. Limerick

The Abbey Bog. Very shallow small bog on carboniferous limestone in S. W. Co. Limerick

Neutral

Ammoniacal Nitrogen evolved expressed as percentage of

Reaction air-dried peat

Neutral

.0079

$.0075 j$

Knockballyboy, King's Co. Carboniferous limestone. Surface peat

Shanagolden, S.W. Co. Limerick. Millstone grit. Altitude, $600-700 \mathrm{ft}$. Surface covered with heather, bog cotton and osmunda

Red bog. Surface, $0^{\prime \prime}-9^{\prime \prime}$ (see above) $\quad \ldots \quad \ldots \quad$ Acid $\left.\quad \begin{array}{l}.0129 \\ .0118\end{array}\right\} B$

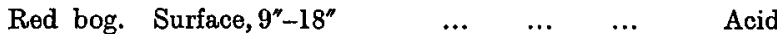

$.0135\} C$

Meeneen, Portumna, Co. Galway. Surface peat...

Acid

$\left.\begin{array}{l}.0235 \\ .0224\end{array}\right\} G$

Scotch Peat:

Corrour, Inverness. Surface peat. Altitude, $1800 \mathrm{ft}$.

Acid

$\left.\begin{array}{l}.0296 \\ .0261 \\ .0260 \\ .0285\end{array}\right\} H$

The amount of ammonia obtained from neutral peats is very much less than that obtained from so called acid peats.

The large quantities of nitrogen obtained in some peats can come from two sources, either from organic compounds capable of hydrolysis by the magnesia, or from ammonia or ammonium salts absorbed by the peat.

It was considered that a qualitative distinction might be made between the two by determining the amount of ammonia which could be removed by extraction with water. The removal of any considerable quantity by water would show that much of it was present adsorbed by the peat.

A suitable quantity of air-dried peat was shaken continuously for 24 hours with ammonia free distilled water and filtered, and an aliquot part distilled with magnesia as before. 
Results of this operation are given in Table IV. In the first column are given amounts of ammoniacal nitrogen expressed as percentages of air-dried peat; in the second column these amounts are expressed as percentages of the amount of ammoniacal nitrogen removed by direct distillation with magnesia:

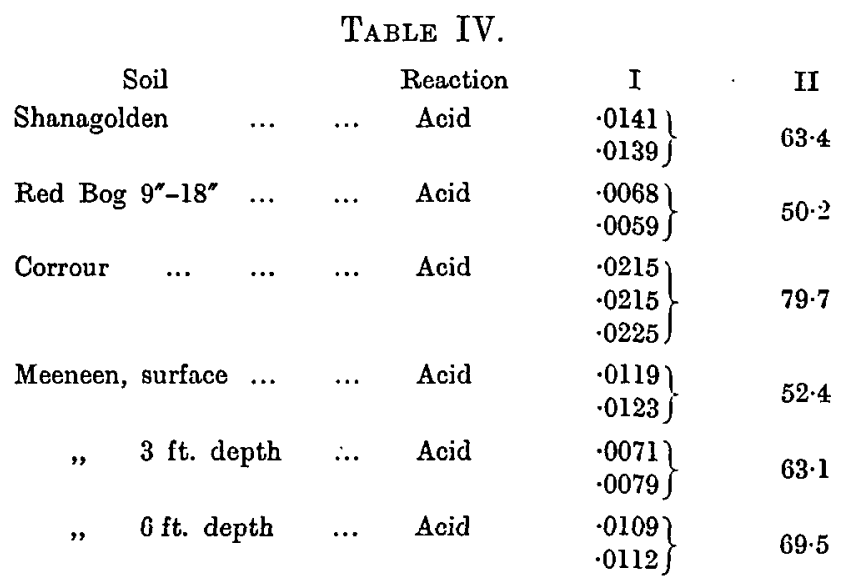

These results are important as showing that the soil water in peat contains ammonia or ammonium compounds which are in equilibrium under the conditions of the experiment with the same substances adsorbed by the colloidal bodies in the humus. Further work is necessary before it is possible to differentiate quantitatively between the nitrogen obtained from adsorbed ammonium compounds and that arising from the decomposition of organic bodies. In Table IV results have been given with peats obtained from 3 feet and 6 feet from the surface as well as from the surface itself, and the figures show that the amounts of ammonia capable of extraction by water differ considerably at the different depths.

This has been found to be a very general phenomenon both in the case of these water extractions and in the case of direct distillations with magnesia. Examples of the latter are given in Table $\mathrm{V}$, which shows the quantities of ammoniacal nitrogen removed by ordinary distillations with magnesia, expressed as percentages of air-dried peats. The results are of 3 soils, and to 3 depths of soil.

The variations observed are great and, as was shown in Table IV, also occur with the amounts of ammonia extracted by water. So far it has been impossible to trace any definite relation between the two sets of figures. 


\section{Ammoniacal Nitrogen of Peats and Humus Soils}

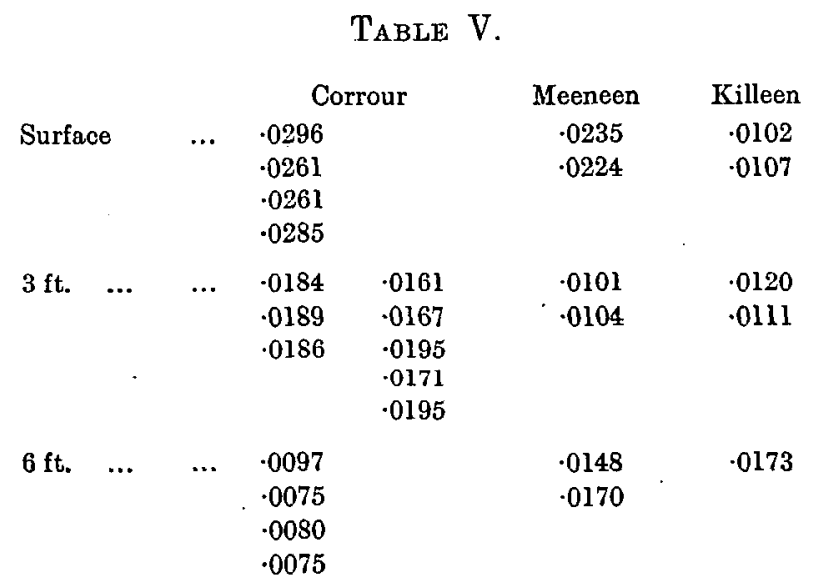

The results of these preliminary investigations may be summarised as follows:

1. Distillation with magnesia under the conditions already described removes an amount of ammonia which is fairly constant for the same peat. This amount is many times greater than in the case of arable soils.

2. Much of the ammonia can be removed by water alone, and no constant ratio has been found between this amount and that obtained by direct distillation.

3. The amounts of ammonia removable by direct distillation with magnesia and by solution in water vary considerably with the depth from which the sample has been obtained.

The nature and source of this ammoniacal nitrogen and the conditions of its solution in the soil water are now being investigated.

This research is being carried out by means of a grant from the Board of Agriculture.

The authors are indebted to $\mathrm{Mr}$ Coyle of the Department of Agriculture and Technical Instruction for Ireland and to Messrs John Kelly and E. J. Delahunty, of the County of Galway and the King's County, respectively, for the collection of some of the samples of Irish peats.

(Received February 2nd, 1916.) 